\title{
La industria maquiladora de Mexicali y aspectos de su funcionamiento
}

Ambar Eugenia Gallegos Arredondo ${ }^{1}$

https://doi.org/10.36105/stx.2018n1.03

\section{Resumen}

Este trabajo muestra la manera en que la industria maquiladora en el estado de Baja California, particularmente en Mexicali, ha sufrido transformaciones a lo largo de los años, y cómo se busca que sean estas empresas las que generen menor costo y mayor producción con mano de obra calificada, por lo que se han tenido que implementar diversos programas de comunicación organizacional interna para que se alcancen objetivos y metas impuestas a inicio de año. También se analizan las fuertes inversiones hechas por parte de gobierno del estado para llevar a cabo grandes campañas y proyectos de promoción que permitan que más empresas extranjeras se instalen en tierras mexicanas, así como el papel que desempeña la responsabilidad social en las organizaciones.

\section{Palabras clave}

Comunicación, industria maquiladora, corporativo, inversión, salario, programas.

\footnotetext{
1 Maestra en Comunicación por la Universidad Autónoma de Baja California, estudiante de tiempo completo del Doctorado de Investigación de la Comunicación (PNPC) en la Universidad Anáhuac México. ambar.gallegosarredondo@gmail.com
} 


\section{El t LC y su impacto en Mexicali}

El mundo de los negocios, de la economía y la manera en que se desarrolla la industria ha venido cambiando desde la firma del Tratado de Libre Comercio de América del Norte (TLCAN), que entró en vigor en 1994. Si bien es cierto que cada uno de los países de esta región tiene un papel individual, existe una fuerte vinculación entre los tres a raíz de este tratado. En particular, en los últimos años éste se ha visto más desarrollado entre México y Estados Unidos, principalmente con los estados que están en la franja fronteriza, así como en los que la industria maquiladora está repuntando.

El TLCAN se creó con la finalidad de que los tres países que lo integran (México, Estados Unidos y Canadá) tuvieran mayor prosperidad, mediante un crecimiento económico derivado de una zona en la que el comercio goza de libre acceso. Pero este tratado no sólo beneficia a la zona comercial, también, en cierto sentido, beneficia al sector industrial, ya que la economía mexicana está liderada por la manufactura; es por esto que, con el crecimiento de México en este sector, su relación con Estados Unidos se está modificando.

Para entrar en contexto, la industria maquiladora se ha convertido en una de las principales fuentes de empleo en Baja California, particularmente en Mexicali, ciudad ubicada en la franja fronteriza con Estados Unidos (estado de California), y que ha permitido que se puedan exportar piezas que son manufacturadas en las empresas de la ciudad y que se ensamblan en Estados Unidos.

A su vez, el TLCAN ha permitido que diversas empresas se instalen en territorio mexicano, una de ellas es Bombardier, empresa canadiense que tiene operaciones en México y Estados Unidos, perteneciente al sector aeroespacial y uno de los principales clientes de algunas empresas instaladas en Mexicali; además, Bombardier es proveedor de servicios de distintas compañías.

Como esta empresa, hay diversidad de compañías que se instalan en el país, particularmente en el estado de Baja California, en donde dan empleo a un gran número de personas, ya sea como operadores o administrativos. La realidad en Mexicali es que la industria maquiladora se ha convertido en una de las principales fuentes de empleo, por lo que es común que los profesionistas al egresar de la universidad busquen una oportunidad en estas organizaciones, con el objetivo de tener estabilidad y un sueldo seguro. En Mexicali se pueden encontrar empresas como Gulfstream, Spectrum, Skyworks, Rockwell Collins, Timsa, SunPower, Newell Rubbermaid, entre otras, las cuales cuentan con oficinas en Estados Unidos y otras partes del mundo, pero que seleccionaron a la ciudad como destino de inversión por la mano de obra y la ubicación. De acuerdo con Montoya, es "La realidad de los impactos económicos y sociales del TLCAN en México, que ha significado la imposibilidad 
de alcanzar altas tasas de crecimiento económico, de generación de empleos y aumento del poder adquisitivo del salario" (2014, p. 15), pues en México el sueldo que se paga tanto a operadores de la industria maquiladora como a administrativos o a profesionistas no tiene comparación con el que se otorga en Estados Unidos. En México un operador de producción cobra 2,200 pesos mensuales mientras que en Estados Unidos un empleado que ejerce esas mismas funciones percibe el equivalente a 45,000 pesos, lo cual es una gran diferencia por un trabajo que se presume se realiza de manera perfecta en el país. Mucho se ha hablado de que México es considerado por la capacidad que tiene la gente para el trabajo, en especial Baja California, que es un estado que cuenta con mano de obra calificada y por esto se promueve como un destino de inversión.

Pero no sólo ocurre esto en el ámbito de producción y con los puestos operativos, sino que, a nivel administrativo y gerencial, las decisiones se manejan desde los corporativos y las personas que ocupan estos puestos en las empresas instaladas en el país rara vez pueden tomar sus propias decisiones. Por lo regular deben consultarlas con el corporativo, incluidas muchas de las autorizaciones en cuanto a presupuesto, y con mayor razón las de comunicación.

\section{La comunicación organizacional interna en las empresas}

En las empresas maquiladoras instaladas en Mexicali la comunicación ha tomado importancia en las organizaciones: "La comunicación es vital para el comportamiento de las organizaciones, los grupos y las personas. En general, la comunicación cumple cuatro funciones básicas en una organización, grupo o persona: control, motivación, expresión de emociones e información" (Chiavenato, 2009, p. 309); a pesar de ello, independientemente de que se tenga un departamento o que se maneje a través de otras áreas, se consulta con el corporativo antes de tomar decisiones relacionadas tanto con la comunicación interna como externa.

Sin embargo, se tiene que reconocer que, ya sea que estén instaladas en Estados Unidos o en México, las necesidades de comunicación de las empresas no son iguales en ambos países, pues se maneja una cultura distinta, un sentido de pertenencia diferente; por esto, la comunicación debería manejarse de manera independiente.

Partiendo de lo anterior, "Toda organización debe considerar a sus integrantes como primer público objetivo: los trabajadores forman un conjunto de individualidades diferenciadas que necesitan información, y su nexo de unión es una relación laboral coordinada que pretende alcanzar un objetivo común” (Franklin \& Krieger, 2011, p. 119). 
En Mexicali, por su situación en la frontera con Estados Unidos, es común ver que se adoptan algunas de las costumbres estadounidenses, pero esto no quiere decir que todo se maneje de la misma manera. En las empresas instaladas en el país se acostumbran ciertos tipos de celebraciones que no se realizan en Estados Unidos; no es la misma cultura y las personas no reaccionan de la misma manera.

Un ejemplo de ello son las fiestas y los convivios; la cultura mexicana es muy dada a la celebración de ocasiones especiales, algo que no es muy común en Estados Unidos, cuya cultura se puede decir que es algo fría o formal, enfocada en realizar su trabajo y cumplir con las metas u objetivos. Aunque es cierto que en Mexicali es común que personas que vienen de fuera comenten que la ciudad parece como si fuera parte de Estados Unidos, que las calles son muy amplias, que los edificios son muy al estilo americano, que celebramos el día de las brujas, el día del pavo, el 4 de julio; que nuestra cultura deja de ser un poco mexicana para convertirse en americana.

En las organizaciones también se maneja una cultura muy parecida a la que se implementa en Estados Unidos, ya que se trabaja bajo una misma visión, misión e incluso valores, que son los que se pactan desde el corporativo, esperando que en el país se alcancen de la misma forma.

También los programas que se implementan para dar reconocimiento y beneficios a los trabajadores son aquellos que marca el corporativo; al final de cuentas, el mismo corporativo es el que autoriza la cantidad de dinero que se invertirá en ello y el periodo en el que se llevará a cabo.

Algo muy común en estas organizaciones es la entrega del mismo producto que maneja la compañía como reconocimiento a los trabajadores, una política creada en Estados Unidos y que se ha adaptado bien en el país, ya que el empleado puede disfrutar de productos que en ocasiones son caros en el mercado y así beneficiarse sin pagar este costo.

En algunas organizaciones no se entregan los productos como "agradecimiento" o "beneficio" para los empleados, sino que se tiene una tienda en la que pueden comprarlos o canjearlos por puntos que van reuniendo y que obtienen al realizar diversas actividades.

Uno de los eventos que se llevan a cabo en algunas empresas de la ciudad y que tienen gran impacto, además de que toman en cuenta la cultura de México, son las celebraciones de cumpleaños, realizadas mes con mes para felicitar a los empleados que cumplen años en ese mes; algunas festejan solamente con un pastel, otras organizan un gran evento con comida y rifa de regalos para los empleados.

Este tipo de eventos, organizados principalmente por el departamento de Recursos Humanos, son muy esperados por los colaboradores. Otro que tiene gran éxito y que es todavía más afín a la cultura mexicana es el evento familiar, el cual se realiza casi siempre en 
el bosque de la ciudad y en el que se ofrece a los empleados, sus parejas e hijos-y en ocasiones también a otros familiares-comida y acceso a las atracciones del parque, así como concursos de disfraces y de canto, y rifa de regalos. Eventos así se han ido multiplicando en las organizaciones porque han sido un éxito. Los empleados los esperan con ansias y se unen a las campañas que hacen las plantas a través de su departamento de Recursos Humanos para que el personal vaya apartando la fecha y esté presente el día del evento.

En algunas organizaciones este evento es temático, por lo que las campañas de comunicación son enfocadas cien por ciento al tema; algunas de las estrategias que se utilizan son carteles en distintos puntos de la planta, videos de promoción con esa temática, así como publicaciones en el órgano de comunicación interna que lo anuncian con anticipación.

\section{Promoción de inversiones a nivel estado}

Otra de las realidades que se vive en Baja California es la fuerte promoción que se realiza principalmente en Estados Unidos, así como en algunos países de Europa, buscando atraer inversiones extranjeras al estado. Con ese objetivo se llevan a cabo diversas giras de promoción en el año.

En el caso específico de Mexicali, la mayoría de estas giras son encabezadas por el gobierno del estado, pero también participa personal de la Secretaría de Desarrollo Económico (Sedeco), de la Comisión de Desarrollo Industrial de Mexicali (CDI), representantes de diversos parques industriales (IAMSA, PIMSA) e incluso representantes de algunas empresas (GKN Aerospace, UTC Aerospace).

Las giras (boletos de avión, hospedaje y viáticos de la comitiva de promoción) se pagan con recursos del estado y tienen una duración aproximada de una semana, aunque algunas pueden alargarse a quince días. El personal que asiste no necesariamente es el que está directamente relacionado con la labor de promoción, lo que implica grandes costos que son pagados con el dinero de los ciudadanos. Un ejemplo del resultado de una gira realizada por el gobernador del estado y la comitiva de promoción se encuentra en Rosillo: "Una inversión de 1 mil 629 millones de pesos para Baja California, equivalente a 90 millones de dólares, es el resultado que trajo el gobernador Francisco Vega de Lamadrid después de reunirse con empresarios de China, Corea del Sur y Japón" (2016).

"La ausencia de la definición de los intereses nacionales, tiene como consecuencia que los objetivos de dicho acuerdo, de promover la inversión productiva y los empleos en los Estados Unidos, vulneren de manera directa las posibilidades de desarrollo económico nacional" (Montoya, 2014, pp. 16-17). Si bien es cierto lo que destaca el autor en esta cita 
textual, también es verdad que el principal objetivo de la promoción que se realiza en Baja California, en especial en Mexicali, con estos eventos de talla internacional, es atraer inversiones. Coincido con el autor en el sentido de que se están desaprovechando estas oportunidades de crecimiento en el país; el recurso público que gasta el gobierno del estado en estas estrategias se podría emplear en desarrollar más las empresas ya instaladas en el país, en especial aquellas pequeñas y medianas empresas que son mexicanas.

También se podría utilizar este recurso para desarrollar los programas dedicados al crecimiento del personal en las empresas ya instaladas en la ciudad; hablando específicamente del área de comunicación, que es el interés de este estudio, se podrían implementar diversos programas para que la comunicación fluya de una mejor manera, una comunicación en la que los empleados tengan una mayor participación, pero sobre todo que a través de la misma se logren los objetivos de la organización.

La comunicación es la base fundamental para el funcionamiento de las empresas, lo difícil es que las organizaciones lo vean de esta manera, que consideren la importancia que tiene y por qué su manejo adecuado puede ser de gran ayuda. Si se invirtiera apropiadamente en programas de comunicación no sólo se estaría beneficiando a los trabajadores, sino también a la misma organización.

\section{La popularidad del outsourcing en las contrataciones}

En el aspecto laboral, en el caso de los trabajadores mexicanos, se debe buscar la manera de brindarles un salario justo, que represente lo que se realiza; particularmente y de suma importancia es que se reconozca que monetariamente tiene el mismo valor que lo que se lleva a cabo en otras plantas, ya que el trabajo es el mismo. La cuestión aquí es que el propio gobierno, el propio país, piensa solamente en sus propios intereses, no en los intereses de las personas, en que los beneficios en cuanto a salarios y prestaciones sean lo mejor para ellos.

Un claro ejemplo de esto es el manejo del outsourcing en el país; cada vez es más común a nivel local que este tipo de empresas den servicio a la industria maquiladora. Años atrás había una sola empresa de outsourcing en la ciudad y hoy en Mexicali se cuenta con 10 o 15, que incluso causan problemas por "robarse" o "piratearse" al personal entre ellas. Para ello se colocan a la salida de los trabajos y ofrecen a los empleados que pasan por ahí un poco más de dinero que su sueldo (en ocasiones pueden ser tan sólo 100 pesos) o mayores prestaciones. Se trata de una manera deshonesta de gestionar el trabajo; lo ideal sería que se tuviera un estándar de sueldo para todas las empresas y que los trabajadores decidieran dónde quieren laborar con base en otro tipo de cuestiones, no sólo por 100 pesos más de sueldo o más 
prestaciones, sino porque la empresa les gusta, porque coincide con su manera de pensar, por sus valores, por su trato; en sí, por cuestiones adicionales a las económicas.

En este aspecto, las empresas deberían operar bajo las normas que rigen al país, no por normas que vienen de Estados Unidos o Europa, que es lo que regularmente pasa, incluso en ocasiones se les ofrece a las empresas algunos descuentos para que se instalen en esas ciudades.

\section{Responsabilidad social en la organización}

Con respecto al medio ambiente, se emplean programas muy similares a los que se aplican en el corporativo: se busca que la empresa maneje de manera correcta sus materiales, que no contamine, que no se desperdicie, incluso hay empresas que otorgan premios a nivel global por el cuidado de estas áreas en la organización, y las plantas buscan obtener este reconocimiento.

Hay otras empresas que lanzan concursos infantiles con esta temática con el objetivo de fomentar la importancia de cuidar el medio ambiente en los niños. Los ganadores reciben premios por su participación e incluso se exponen sus dibujos; esto se logra con campañas de comunicación organizacional interna, tanto para informar las bases de la convocatoria, las fechas en las que se seleccionarán los ganadores, como para anunciar a los colaboradores la fecha en que tendrá lugar el evento de premiación. Para Rebeil (2012)

Uno de los retos más relevantes que la Responsabilidad Social Organizacional enfrenta en México es el hecho de evitar al máximo que la mayoría de las organizaciones que emprendan procesos de RSO lo vean como una mera oportunidad de marketing social o una forma más para mejorar su imagen y su reputación. (p. 21)

\section{Instalación de empresas extranjeras}

Hoy en día México es volteado a ver por la mano de obra calificada y barata con la que cuenta. Hubo un caso hace unos siete años en Mexicali en el que una empresa que tenía bastante tiempo en la ciudad cerró sus instalaciones para trasladarse a Tamaulipas, donde la mano de obra operativa era más económica; lo que no esperaban era que no funcionara la estrategia y que tuvieran que regresar a Mexicali porque en Tamaulipas la calidad que requería el producto no era la necesaria. El problema aquí es que no sólo se busca el beneficio económico 
fuera del país, sino también dentro del mismo; aquí, de nueva cuenta, no se está pensando en respetar a la gente, sino en buscar el beneficio propio.

Otros casos son los de las empresas que cierran sus instalaciones en México para abrir operaciones en China, porque esto representa un gran ahorro en el proceso de producción, ya que en ocasiones se les paga a los empleados la mitad de lo que se paga en México; como lo importante es ahorrar costos, prefieren cerrar operaciones e irse a otro país.

A pesar de ello, para el gobierno de Baja California una de sus tareas principales ha sido la promoción de inversiones extranjeras en el estado, siendo una de sus prioridades las empresas del sector aeroespacial, uno de los sectores más fuertes en el estado, ya que diversas empresas de este giro se han instalado aquí y han ido creciendo; tal parece que Mexicali se ha convertido en una de las principales ciudades para desarrollar ese trabajo. El interés por que se establezcan empresas en la ciudad es muy grande, así que en ocasiones se otorgan beneficios a las organizaciones para que se queden aquí.

Un claro ejemplo de ello es la instalación de una empresa de este giro en la ciudad: "La mañana de este martes, se instaló en Mexicali la empresa Barnes Aerospace, que realizará la manufactura de componentes estructurales, partes para turbina con base de aluminio, acero y materiales compuestos" (Heras, 2016).

Sin embargo, uno de los riesgos de que se estén instalando tantas empresas que trabajan con las normas del corporativo es la pérdida de la identidad: que se trabaje bajo una cultura organizacional que no va de acuerdo con la cultura del país, enfocada de manera principal en el alcance de la producción. De ahí la importancia de que se considere la comunicación como una base para el desarrollo de los programas que ahí se manejan, para que el empleado se sienta cómodo y no como un robot que sólo trabaja para lograr la cuota de producción diaria.

Sería interesante analizar cuáles son los beneficios que dejan las empresas extranjeras en el país. En Mexicali la realidad es que la industria maquiladora es una de las principales fuentes de empleo; cabría preguntarse qué pasaría si no hubiera industria maquiladora, ¿en qué trabajaría la gente? Si esto pasara en realidad, estaría bien conocer si las personas se sentirían atraídas hacia la innovación, hacia la creación de empresas; si con unas políticas distintas tendrían la posibilidad de desarrollarse las pequeñas y grandes empresas y si podría aportar a la economía y al crecimiento del país que se inculcara a las personas, desde temprana edad, que la inversión propia y la creación de empresas propias puede representar una gran oportunidad, y no solamente el escalar en la organización, donde el sueño es irse a trabajar a las oficinas del corporativo. 


\section{Diversidad de programas en las organizaciones}

Es cierto que en la industria maquiladora se apoya a los empleados para que se preparen: algunas empresas tienen programas para que los empleados (principalmente operadores) puedan terminar su educación básica; y también hay programas de inglés en otras, así como apoyos para terminar la licenciatura y para el estudio de diplomados, especialidades y maestrías.

Esta cuestión parece no pasar del todo inadvertida para las empresas que están apoyando con estos programas la preparación y el crecimiento profesional de sus trabajadores; lo que sería de mayor ayuda es que se empezaran a brindar mayores oportunidades de crecimiento para quienes busquen seguir estudiando y aprendiendo.

Un punto desarrollado recientemente en las empresas, que viene desde el corporativo, es el que busca que las cosas se hagan con menos; en este caso se pide a un empleado que haga más actividades por el mismo sueldo y en ocasiones éste termina haciendo las actividades de dos personas y percibiendo el pago de una; lo llaman reingeniería, pero podría interpretarse como un tipo de explotación. Las personas aceptan la nueva carga de trabajo porque necesitan el sueldo para vivir, pero no parece justo que para obtener el pago se tenga que llegar a ese punto. En este sentido se podría buscar un acuerdo con las empresas para que al momento en que tengan crecimiento el país se beneficie con ello, y se pudieran poner en marcha programas específicos de apoyo a la economía del país y en beneficio de la comunidad; así, el hecho de que se conviertan en potencia redundaría en el bien del país. Para Montoya (2012),

Se debe rediseñar una nueva economía política sustentada en el mercado interno, un nuevo pacto entre salarios y utilidades y un acto fiscal integral, dirigido a la finalidad de que México sea potencia económica por sus propios méritos y no por ser lugar de paso de las inversiones y el comercio de las corporaciones globales, con claros criterios de equidad social y soberanía ante la globalización. (p. 21).

Si se lograra esta modificación en la que se beneficiara al país, se podría alcanzar un gran desarrollo económico e incluso social y se podrían ofrecer muchas más prestaciones y beneficios a las personas. Las inversiones que se tienen en el país son muy buenas, pues dan empleo a muchas personas, en especial en Baja California, pero si se viera la manera de beneficiar a los empleados sería mucho mejor, ya que se alcanzaría un nivel de satisfacción superior.

En este punto es clave señalar que representantes de algunas empresas hacen mención de que el gobierno del estado se debe preocupar más por mantener a las empresas que ya están 
instaladas en la ciudad en vez de efectuar giras de promoción y seguir buscando inversiones; que más bien se deben procurar apoyos para estas empresas, pues se ha visto complicada la situación económica y esto afecta la creación de empleos.

Una de las gerentes generales de una empresa del sector textil refiere su encuentro con el gobernador del estado (Baja California), quien regresaba de una gira de promoción en Asia y muy orgulloso le dijo que se instalarían varias empresas en la ciudad en este año. Ella le respondió que lo mejor sería que dejara de hacer giras y se enfocara en apoyar a las empresas que ya están instaladas.

Es una realidad que se seguirán realizando estas giras de promoción en Baja California; son uno de los principales objetivos tanto de los gobernadores como del presidente municipal (al menos el de la capital del estado) y representan metas que se proponen cumplir durante su periodo. Si bien es cierto que también fijan un número para retención de empresas, la mayor parte del presupuesto se enfoca en estas giras de promoción.

También es cierto que mientras se sigan instalando empresas extranjeras en nuestro país, los beneficios seguirán siendo para ellos, por eso la importancia de que se busquen dentro de las organizaciones programas que beneficien a los empleados, que tengan como prioridad su bienestar: un empleado que se siente valorado y motivado puede aportar mucho a la organización.

\section{Referencias}

Chiavenato, I. (2009). Comportamiento organizacional. La dinámica del éxito en las organizaciones. México: Mc Graw Hill.

Franklin, E. \& Krieger, M. (2011). Comportamiento organizacional. Enfoque para América Latina. México: Pearson.

Gobierno de Canadá (2012). División de transporte de Bombardier: “Por buen camino...”. junio 18, 2016. Recuperado de TLCANHOY.ORG http://www.tlcanhoy.org/success/canada_es.asp

Heras, L. (13 de julio de 2016). Con 8 mdd de inversión, se instala en Mexicali la empresa Barnes Aerospace.La fornada Baja California. Recuperado de http://jornadabc.mx/tijuana/07-06-2016/ con-8-mdd-de-inversion-se-instala-en-mexicali-la-empresa-barnes-aerospace

Montoya, A. (18 de septiembre de 2014). Análisis del II Informe entregado al Congreso de la Unión por Enrique Peña Nieto, Presidente Constitucional de los Estados Unidos Mexicanos, en materia de Política Exterior. Comparecencia ante el H. Senado de la República del Secretario de Relaciones Exteriores José Antonio Meade Kuribreña. Mimeo.

Montoya, A. (2012). El rescate de la soberanía nacional. En E. Mendoza (Coord.), Los retos de México (pp. 63-88). México. 
Montoya, A. (2012). Soberanía y desarrollo. En Ricardo Valero (Coord.), 2010: Reforma del Estado y fortalecimiento de la nación (pp. 213-236). México, D. F.: UNAM.

Rebeil, M. (2012). Responsabilidad social organizacional. México: Trillas.

Rosillo, R. (13 de julio de 2016). Confirma Sedeco 90 millones de dólares de inversión para BC. El Mexicano. Recuperado de: http://www.el-mexicano.com.mx/informacion/noticias/1/3/ estatal/2016/03/29/954752/confirma-sedeco-90-millones-de-dolares-de-inversion-para-bc 